\title{
Predicting Optimum Work Force for a Tool and Cutter Grinding Machine using Posture Analysis
}

\author{
G Madhan Mohan ${ }^{1}, \mathrm{C}$ Vigneswaran ${ }^{2}$ \\ \{gmm.prod@psgtech.ac.in ${ }^{1}$, cvn.prod@psgtech.ac.in ${ }^{2}$ \}
}

\author{
Professor (CAS), Department of Production Engineering, PSG College of Technology, \\ Coimbatore, India ${ }^{1}$, Assistant Professor (Sr. Gr), Department of Production Engineering, PSG \\ College of Technology, Coimbatore, India ${ }^{2}$
}

\begin{abstract}
In India, small and medium-scale industries depend on traditional conventional machine tools for their manufacturing activities. Amongst them, 'Tool and cutter grinding machine' is economically significant and also a bottleneck machine. The machine feeds cutting tools and cutters for other machine tools after completion of the re-grinding process. The Absenteeism of an operator in the machine affects the industry productivity. The study analyzes the reasons for absenteeism and its remedial actions. Among various reasons stated by operators of the machine, fatigue draws critical attention and scope for an ergonomic study. The novelty of this work is to determine the optimal anthropometric dimensions of the workforce that can operate the tool and cutter grinder machine without absenteeism and less fatigue. Therefore, work-study has been performed to identify repetitive upper limb movements of the operators during machine operations. The observation results call for a postural investigation. Rapid Upper Limb Assessment method (RULA), a popular upper limb postural assessment method, has been selected for the investigation. 35 operators from a small-scale industry have been inducted for the investigation. Anthropometric data were collected from the operators. Digital Human Models (DHM) have been built for all the postures occupied by the operators, to assess the RULA score. The anthropometric data and RULA score have been correlated to predict the optimal population with less fatigue using the regression equation. The optimum range of anthropometric data to operate the machine was determined based on regression analysis.
\end{abstract}

Keywords: Work posture, RULA, DHM, Tool and cutter grinder, work fatigue, regression analysis.

\section{Introduction}

This This Micro, Small, and Medium Enterprises (MSME) contribute nearly one-third of India's Gross Domestic Product (GDP). It provides more than ten crore jobs, of which 30\% comes from the manufacturing segment alone [10]. The segment comprises casting, plastic molding, forming, machining, join- ing, heat treatment, surface treatment, and powder metallurgy jobs [12]. Among them, machining is one of the traditional jobs, involving machine tools and metal cutting operations. In India, machine tools used by MSMEs are conventional, although automated versions are available at a high cost. Metal cutting operations in these machine tools are performed mostly through re-grindable cutting tools, although disposable tools are available at a high cost. In a single-point cutting tool: six cutting 
edges including the rake angle influences the mechanics of chip formation. Other cutting tools used in drilling and milling further have a complex nomenclature, making re-grinding more complex [13]. Considering the complex nomenclature, these traditional cutting tools might lose their cutting ability after its specified tool life period, which further demands a replacement of a new tool. MSMEs in India prefer to regrind the tools nomenclature as they are less affordable and lack the need to buy a new tool frequently for their production purpose. Even though re-grinding of the tools are complex, it is still in practice and inevitable.

For the said requirement, normal bench grinders would not suit the purpose. The regrind of cutting edges and angles for a lathe tool and milling cutters need a more precise setup. These re-grindable tools decide the performance of the machining operation, and so regrinding cutting edges is done in a tool and cutter grinding machine. Therefore, the machine becomes a bottleneck operation in an industry as other machine tools depend on the output of this machine.

Tool and cutter grinding is one of the traditional machines that, would suit the grinding operation of worn-out cutting tools. The re-grinding operation requires both the hands of a semi-skilled operator to get involved. Elaborately to say, right- hand wrist to feed the cutting tool and left-hand wrist to give a depth of cut. In Figure 1, a sample tool and cutter grinder, with a vice setup, is shown to facilitate the readers' visualization. The machine has more controls and working levers to support the re-grinding operation. During operation, the machine demands different occupational postures of the worker to complete the given re-grinding task. Hence, continuously working on the machine would be tiresome and may cause Musculoskeletal Disorders (MSDs). The worker prone to MSDs would affect his / her health, further leading to absenteeism [14] and loss of man-hour. This makes the bottleneck operation more crucial in MSMEs. As the worker occupies more postures, ergonomic studies have been initiated to bring down fatigue of the workers and hence absenteeism.

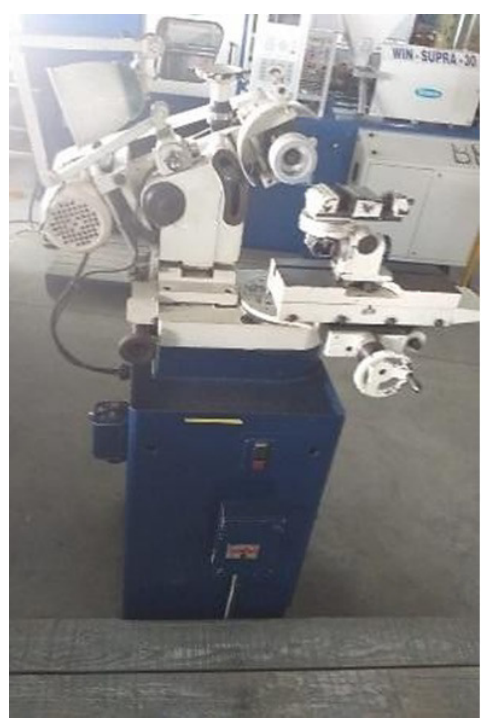

Fig. 1. Photographic Image of the Tool and Cutter Grinding Machine 
Literature related to working postures, virtual ergonomic assessment using Digital Human Modeling (DHM) are dis- cussed in this section. Ergonomics provides information on human behavior, abilities, limitations, and other characteristics to the design of tools, machines, tasks, jobs, and environments for productive, safe, comfortable, and effective human use. Several factors play a role in ergonomics like body posture and movement (sitting, standing, lifting, pulling, and pushing). The goal of ergonomics is to provide maximum productivity with minimal effort by the application of ergonomic principles. Hence, people should be able to perform the job without the risk of injury [9]. Ergonomics has its primary focus on the design of work activity that suits the person's capabilities and limitations. Working posture and working time duration are the main reasons for muscular ailments [5]. Rapid Upper Limb Assessment (RULA) is a tool used to identify the postural score [11]. RULA has been practiced to assess the fatiguing nature of the repeating postures [7]. The ergonomic assessment was done both manually and in a CAD environment [2] for the accuracy and precision of the results.

Computer Aided Design (CAD) and Digital Human Modeling (DHM) (specialized CAD software for virtual human representation) technologies endow unique opportunities to incorporate human factors pro-actively in design and development [16]. Digital Human Modeling (DHM) is one of the dynamic virtual simulation techniques in the field of ergonomic assessment [15]. The spinal posture of the worker could be an influential factor of discomfort during the given task [4].

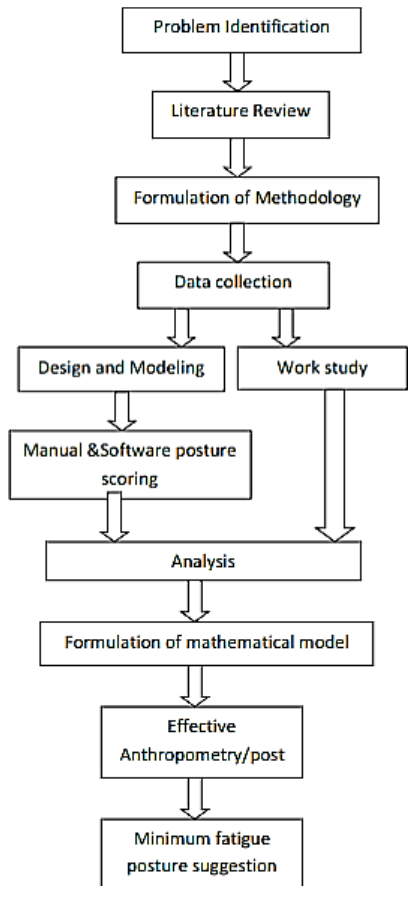

Fig. 2.Methodology Flow Chart 
A DHM has been developed by considering the parameters like neck flexion, shoulder abduction, elbow pronation, wrist ulnar deviation [3]. The developed DHM was considered for virtual postural investigation using a CAD environment [6].From the literature survey, it is evident that postural assessment of a worker, using a CAD environment would be more effective without demanding real-time enactments [11]. The impressions from the literature motivated the authors to carry out the virtual postural assessment of a worker to predict an optimum anthropomorphic workman height range to operate the machine with a minimum fatigue. The main objective of the work is to reduce the Musculoskeletal Disorders [1] during tool and cutter grinding operation by assessing the existing work posture. Hence to satiate the said objective, a case study in a small scale manufacturing industry was conducted.

\section{Methods:}

As a criterion for the DHM based evaluation, all the 35 subject samples were ensured with good health. The subjects were investigated for muscular diseases of any sort. The samples clearly explained the procedure in a relaxed condition. A methodology has been framed as shown in Figure 2. Vital physical measures like stature (height) were noted along with other anthropometric parameters. Acromion-radiale (Elbow to wrist), wrist to fingertip, radiale-stylion (Shoulder to elbow), waist height (Hip to foot) and hand length were tabulated

The work-study[8] has been carried out while the workers were individually performing a single-point cutting tool grinding task. The task has been performed to develop a basic understanding of work nature. The critical aspects involved during the operation were witnessed. The observation of the work was video graphed (Figure 3) using a digital camera. The recorded video was further split into smaller elements for ease of analysis.

Based on the motion of the worker, each activity was categorized into a particular therblig symbol. The observations were recorded using a two-handed chart (Table 1). From the workstudy, different work postures of the worker during the machine operation were observed. The posture that repeated again and again has been taken into consideration for further analysis.

The obtained work-study data was used to analyze the fatigue prone posture of the worker. Physical working posture has been photographed during the operation. The photographs were closely analyzed to quantify worker fatigue using RULA score.

Together, DHM has been performed with the collected human anthropometry data from the sample. The obtained data is discontinuous; therefore, the perfect range of anthropometry remained unable to figure it out. Hence, a simple linear regres- sion model has been formulated to obtain continuous data. The anthropometry data of the human model was customized in a CAD environment with the predicted regression model. The normal distribution bell curve was utilized to segregate the population in percentile.

The formation of a simple linear regression model was used to evaluate the fatigue of the worker. In this model, height was considered as an independent variable to predict the dependent variables like waist height (Table 3) and hand length (Table 4). The continuous data points for waist height and hand length have been found from the regression equations (Table 5). The bell curve was plotted to distinguish the percentile within the continuous data. The 
predicted values of hand length and waist height data from Table 5 was used for Digital human modeling in CAD environment.

\section{Results And Discussions}

The appropriate worker's compatibility has been validated by analyzing the photographs with the manikin generation. The results for various percentile values have been obtained from the virtual ergonomic postural assessment. The assessments indicate that the RULA score was low and unchanged for the values between 0th and the 50th percentile. Alternatively, the RULA score has increased after $50^{\text {th }}$ percentile value. From Figure. 4 it is apparent that the RULA score has been observed as 7 for the 95th percentile model. The numerical value obtained through the RULA score clinches the fatiguing nature experienced by the worker. The obtained experimental data has been cross-verified using both DHM and manual evaluation. The assessment clearly shows that the operator's height range is a major influential parameter while working on a tool and cutter grinding machine. An increase in the score is majorly caused by the posture variation due to an increase in height. This increase in height causes a more bending angle in the lumbar region; this would lead to an increase in fatigue to the worker. Also, the appropriate height range adapted for the given machine tool would minimize the operator's fatigue. Less fatigue would reduce absenteeism and loss of man-hour in the machinery. The height range that an operator could work in this machine with less fatigue is found to be between $153 \mathrm{~cm}$ and $174 \mathrm{~cm}$.

\section{Conclusion}

This paper considers the dependency of traditional conventional machines used by small and medium scale manufacturing industries in India. The workers in small-scale industries experience fatigue during the machining operation. This was the inspiration to perform the experimental task in a small scale industry, situated at Coimbatore. A detailed experimental study has been carried out as per the methodology planned. The observations from the study claim to select a particular machine with worker absenteeism. Therefore, the tool and cutter grinding machine has been selected. A complete analysis has been performed to understand the basic operation and root cause of absenteeism. It shows the existing work posture of a worker, working in a tool and cutter grinding machine causes work fatigue. The existing work posture has been analyzed using DHM. DHMs' have been constructed for the data obtained from the regression models. The evaluation of DHMs' determines the perfect population, experiencing minimal fatigue in this particular machine. From the results and discussion section, the predicted optimal height range of an operator has been found between $153 \mathrm{~cm}$ and $174 \mathrm{~cm}$.

On the other hand, this experiment would also support the ability to select and suit a skilled person with an optimal height range for the required task. The objective of this work is to reduce human fatigue by predicting the optimal height range of an operator. Further, this prediction would serve as an input to identify the work-aid supplements required for an operator tobe used as a template for papers to be published in EAI Core Proceedings. Follow the text for further instructions on text formating, tables, figures, citations and references. 


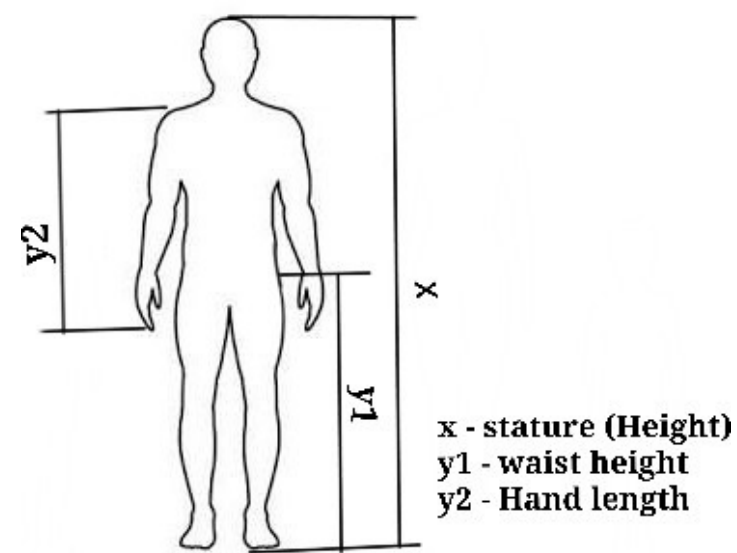

Fig. 3. Anthropometric Parameters

TABLE I

Two- HANDEd ChaRT (WoRk-STUdy)

$\begin{array}{llll}\text { Left-hand Description } & \text { Symbol } & \text { Right-hand Description } \\ \text { Search for tool and handle } & \text { Sh } & \text { Sh } & \text { Search for tool and handle } \\ \text { Grasp the tool } & \text { G } & \text { G } & \text { Grasp the tool } \\ \text { Position the tool } & \text { P } & \text { G } & \text { Grasp the handle } \\ \text { Hold the vice } & \text { H } & \text { A } & \text { Adjust the vice } \\ \text { Search for spanner } & \text { Sh } & \text { Sh } & \text { Search for spanner } \\ \text { Hold the vice } & \text { H } & \text { G } & \text { Grasp the spanner } \\ \text { Hold the vice } & \text { H } & \text { DA } & \text { Loosen the nut } \\ \text { Adjust the position of vice } & \text { P } & \text { A } & \text { Tighten the nut } \\ \text { Hold the vice } & \text { H } & \text { DA } & \text { Loosen the nut } \\ \text { Adjust the position of vice } & \text { P } & \text { A } & \text { Tighten the nut } \\ \text { Grasp the handle } & \text { G } & \text { G } & \text { Grasp the lever } \\ \text { Move the tool close to the wheel } & \text { U } & \text { G } & \text { Grasp the lever } \\ \text { Apply load gradually } & \text { U } & \text { U } & \text { Move the lever back and forth } \\ \text { Move the tool away from the wheel } & \text { U } & \text { AD } & \text { Rest } \\ \text { Hold the vice } & \text { H } & \text { G } & \text { Grasp the spanner } \\ \text { Hold the vice } & \text { H } & \text { DA } & \text { Loosen the nut } \\ \text { Adjust the position of vice } & \text { P } & \text { A } & \text { Tighten the nut } \\ \text { Grasp the handle } & \text { G } & \text { G } & \text { Grasp the lever } \\ \text { Move the tool close to the wheel } & \text { U } & \text { G } & \text { Grasp the lever } \\ \text { Apply load gradually } & \text { U } & \text { U } & \text { Move the lever back and forth } \\ \text { Move the tool away from the wheel } & \text { U } & \text { AD } & \text { Rest } \\ \text { Grasp the wheel in the left side } & \text { G } & \text { AD } & \text { Rest } \\ \text { Adjust the wheel } & \text { U } & \text { AD } & \text { Rest } \\ \text { Grasp the handle } & \text { G } & \text { G } & \text { Grasp the lever } \\ \text { Move the tool close to the wheel } & \text { U } & \text { G } & \text { Grasp the lever } \\ \text { Apply load gradually } & \text { U } & \text { U } & \text { Move the lever back and forth } \\ \text { Move the tool away from the wheel } & \text { U } & \text { AD } & \text { Rest } \\ \text { Hold the vice } & \text { H } & \text { G } & \text { Grasp the handle } \\ \text { Hold the vice } & \text { H } & \text { DA } & \text { Loosen the vice }\end{array}$




\begin{tabular}{llll}
\hline Population size $(\mathrm{n})=35$ Nos & Stature $($ Height $)(\mathrm{x})$ & Waist height $($ Hip to foot $)\left(\mathrm{y}_{1}\right)$ & Hand length $\left(\mathrm{y}_{2}\right)$ \\
Mean & 170.428 & 99.428 & 75.257 \\
SD & 8.503 & 5.847 & 5.198 \\
Max & 188 & 112 & 89 \\
Min & 153 & 89 & 65 \\
\hline
\end{tabular}

TABLE IIIREGRESSION MODEL FOR WAIST HEIGHT

\begin{tabular}{|c|c|c|c|}
\hline $\begin{array}{l}\text { Description } \\
\text { Sum of height }\end{array}$ & $\begin{array}{l}\text { Symbol } \\
\varnothing R x\end{array}$ & Values & Formula \\
\hline Sum of waist height & $\varnothing \mathbf{R ́}_{1}$ & 3480 & $\mathbf{n}_{1} \mathrm{y}_{1}$ \\
\hline Average of height & $\mathrm{X}$ & 170.42 & $\mathrm{x} / \mathrm{n}$ \\
\hline Average of waist height & $\mathrm{Y}_{1}$ & 99.42 & $\mathrm{y}_{1} / \mathrm{n}$ \\
\hline Sum of the square of $x$ & $\operatorname{ssxx}$ & 2458.57 & $(x-X)^{2}$ \\
\hline Sum of the square of $y$ & ssyy $_{1}$ & 1162.57 & $\left(\mathrm{y}_{1}-\mathrm{Y}_{1}\right)^{2}$ \\
\hline Sum of x.y & $\mathbf{s s x y}_{1}$ & & \\
\hline Correlation & $\mathrm{r}$ & 0.7778 & $\operatorname{Ssxy}_{1} /\left(\operatorname{ssxx}^{*} \operatorname{ssyy}_{1}\right)$ \\
\hline Constant & $b_{1}$ & 0.605 & ssxy/ssxx \\
\hline Constant & $b_{0}$ & -3.692 & $\mathrm{Y}_{1}-\mathrm{b}_{1} \mathrm{X}$ \\
\hline
\end{tabular}

TABLE IVREGRESSION MODEL FOR HAND LENGTH

\begin{tabular}{|c|c|c|c|}
\hline $\begin{array}{l}\text { Description } \\
\text { Sum of height }\end{array}$ & $\begin{array}{l}\text { Symbol } \\
\text { Øর́x }\end{array}$ & Values & Formula \\
\hline Sum of waist height & $\varnothing \mathrm{Ŕ}_{2}$ & 2634 & $\mathbf{n}_{1} \mathrm{y}_{2}$ \\
\hline Average of height & $X$ & 170.42 & $\mathrm{x} / \mathrm{n}$ \\
\hline Average of waist height & $\mathrm{Y}_{2}$ & 75.257 & $\mathrm{Y} 2 / \mathrm{n}$ \\
\hline Sum of the square of $x$ & $\operatorname{ssxx}$ & 2458.57 & $(x-X)^{2}$ \\
\hline Sum of the square of $y$ & $\mathrm{Ssyy}_{2}$ & 918.686 & $\left(y_{2}-Y_{2}\right)^{2}$ \\
\hline Sum of $x . y$ & $\mathrm{Ssxy}_{2}$ & & \\
\hline Correlation & $\mathrm{r}$ & 0.807 & $\mathrm{Ssxy}_{2} /\left(\operatorname{ssxx}^{*} \mathrm{ssyy}_{2}\right)$ \\
\hline Constant & $\mathrm{b}_{1}$ & 0.493 & ssxy/ssxx \\
\hline Constant & $\mathrm{b}_{0}$ & -8.76 & $\mathrm{Y}_{2}-\mathrm{b}_{1} \mathrm{X}$ \\
\hline
\end{tabular}

perform a fatigue less task. In this case, an autonomous height adjustment was suggested to the management of the targeted small scale industry. However, the work was performed to facilitate an anthropometric deficit worker with work aid supplements and not to eliminate from the employment.

At this height range, there is a positive sign that there is a scope of reduction in standard time due to lower fatigue allowance value. Also, like the human height range prediction for a particular machine, the other vital human anthropometric data could be mapped for a given machining task.

Acknowledgements

Authors would like to thank the support of the

Industrial Institute, Coimbatore, India

management, staff, and workers of PSG 
Conflict Of Interest

The authors declare that they have no conflict of interest.

\section{References}

[1] D. Lämkull, L. Hanson, and R. Örtengren, "A comparative study of digital human modelling simulation results and their outcomes in reality:A case study within manual assembly of automobiles," International Journal of Industrial Ergonomics, vol. 39, no. 2, pp. 428-441, 2009.

[2] M. Mazzola, L. Forzoni, S. D\&apos;onofrio, and G. Andreoni, "Use of digital human model for ultrasound system design: a case study to minimize the risks of musculoskeletal disorders," International Journal of Industrial Ergonomics, vol. 60, pp. 35-46, 2017.

[3] A. Naddeo, N. Cappetti, and C. Oria, "Proposal of a new quantitative method for postural comfort evaluation," International Journal of Indus- trial Ergonomics, vol. 48, pp. 25-35, 2015.

[4] A. Naddeo, R. Califano, M. Vallone, A. Cicalese, C. Coccaro, F. Mar- cone, and E. Shullazi, "The effect of spine discomfort on the overall postural (dis) comfort," Applied ergonomics, vol. 74, pp. 194-205, 2019.

[5] J. Sanjog, T. Patel, A. Chowdhury, and S. Karmakar, "Musculoskeletal ailments in Indian injection-molded plastic furniture manufacturing shop-floor: Mediating role of work shift duration," International Journal of Industrial Ergonomics, vol. 48, pp. 89-98, 2015.

[6] F. Demoly and S. Roth, "Knowledge-based parametric CAD models of configurable biomechanical structures using geometric skeletons," Computers in Industry, vol. 92, pp. 104 $117,2017$.

[7] B. D. Lowe, P. G. Dempsey, and E. M. Jones, "Ergonomics assess- ment methods used by ergonomics professionals," Applied ergonomics, vol. 81, pp. 102 882-102 882, 2019.

[8] ShyamBhatawdekar, K. Dr, and Bhatawdekar, pp. 5-11, 2012.

[9] R. Bridger, Introduction to Ergonomics2008.

[10] 2018. [Online]. Available: https://msme.gov.in/relatedlinks/annual- report-ministry-micro-smalland-medium-enterprises

[11] G. M. Mohan, P. S. S. Prasad, and V. Sudarmathi, "Simplified procedure to analyze work posture of foundry men through the Rapid Upper LimbAssessment," International Journal of Environment and Health, vol. 2, no. 2, 2008.

[12]Z. Huda, "Materials Processing for Engineering Manufacture," Founda- tions of Materials Science and Engineering, 2016.

[13] R. Komanduri, M. Lee, and L. M. Raff, "The significance of normal rake in oblique machining," International Journal of Machine Tools and Manufacture, vol. 44, pp. 1115-1124, 2004.

[14] R. D. Hacket, "Work attitudes and employee absenteeism: A synthesis of the literature," Journal of occupational psychology, vol. 62, pp. 235-248, 1989.

[15] M. Charu, M. Karmakar, S. Das, and A, "Digital human modeling (DHM) for improving work environment for specially-abled and el- derly," SN Applied Sciences, vol. 1, pp. 1326-1326, 2019.

[16] T. Patel, J. Sanjog, and S. Karmakar, "Ergonomics Perspective in Agri- cultural Research: A User-Centred Approach Using CAD and Digital Human Modeling (DHM) Technologies 
TABLE V

[17] Predicted data From Regression Model

\begin{tabular}{|c|c|c|}
\hline Stature & Waist height & Hand length \\
\hline 150 & 87.06 & 65.181 \\
\hline 151 & 87.665 & 65.674 \\
\hline 152 & 88.27 & 66.167 \\
\hline 153 & 88.875 & 66.66 \\
\hline 154 & 89.48 & 67.153 \\
\hline 155 & 90.085 & 67.646 \\
\hline 156 & 90.69 & 68.139 \\
\hline 157 & 91.295 & 68.632 \\
\hline 158 & 91.9 & 69.125 \\
\hline 159 & 92.505 & 69.618 \\
\hline 160 & 93.11 & 70.111 \\
\hline 161 & 93.715 & 70.604 \\
\hline 162 & 94.32 & 71.097 \\
\hline 163 & 94.925 & 71.59 \\
\hline 164 & 95.53 & 72.083 \\
\hline 165 & 96.135 & 72.576 \\
\hline 166 & 96.74 & 73.069 \\
\hline 167 & 97.345 & 73.562 \\
\hline 168 & 97.95 & 74.055 \\
\hline 169 & 98.555 & 74.548 \\
\hline 170 & 99.16 & 75.041 \\
\hline 171 & 99.765 & 75.534 \\
\hline 172 & 100.37 & 76.027 \\
\hline 173 & 100.975 & 76.52 \\
\hline 174 & 101.58 & 77.013 \\
\hline 175 & 102.185 & 77.506 \\
\hline 176 & 102.79 & 77.999 \\
\hline 177 & 103.395 & 78.492 \\
\hline 178 & 104 & 78.985 \\
\hline 179 & 104.605 & 79.478 \\
\hline 180 & 105.21 & 79.971 \\
\hline 181 & 105.815 & 80.464 \\
\hline 182 & 106.42 & 80.957 \\
\hline 183 & 107.025 & 81.45 \\
\hline 184 & 107.63 & 81.943 \\
\hline 185 & 108.235 & 82.436 \\
\hline 186 & 108.84 & 82.929 \\
\hline 187 & 109.445 & 83.422 \\
\hline 188 & 110.05 & 83.915 \\
\hline 189 & 110.655 & 84.408 \\
\hline 190 & 111.26 & 84.901 \\
\hline 191 & 111.865 & 85.394 \\
\hline 192 & 112.47 & 85.887 \\
\hline 193 & 113.075 & 86.38 \\
\hline 194 & 113.68 & 86.873 \\
\hline 195 & 114.285 & 87.366 \\
\hline 196 & 114.89 & 87.859 \\
\hline 197 & 115.495 & 88.352 \\
\hline 198 & 116.1 & 88.845 \\
\hline 199 & 116.705 & 89.338 \\
\hline 200 & 117.31 & 89.831 \\
\hline 201 & 117.915 & 90.324 \\
\hline 202 & 118.52 & 90.817 \\
\hline 203 & 119.125 & 91.31 \\
\hline
\end{tabular}




$\begin{array}{lll}204 & 119.73 & 91.803 \\ 205 & 120.335 & 92.296 \\ 206 & 120.94 & 92.789 \\ 207 & 121.545 & 93.282 \\ 208 & 122.15 & 93.775 \\ 209 & 122.755 & 94.268\end{array}$

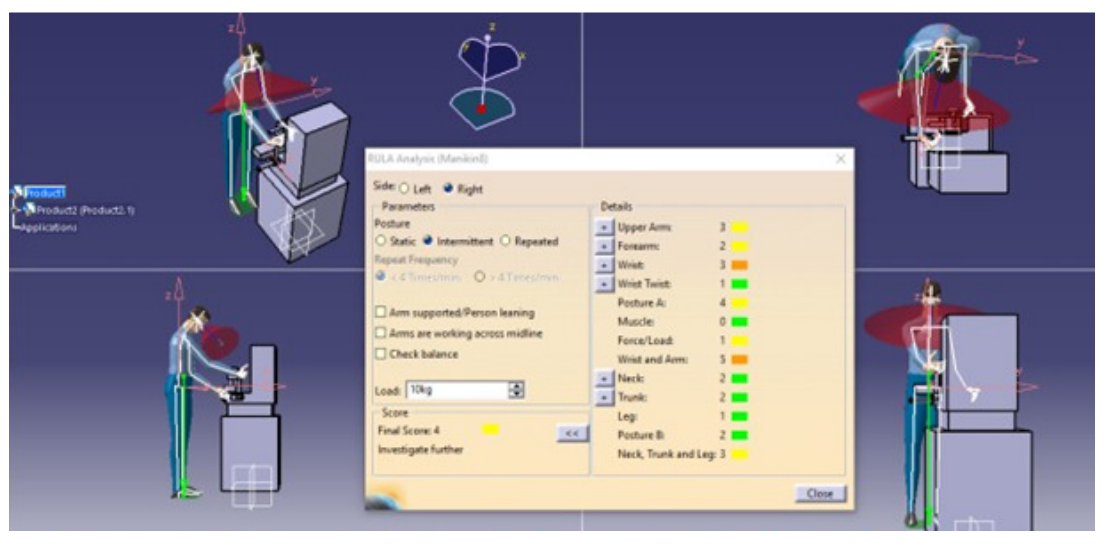

Fig. 4. 50th Percentile DHM \& RULA Scoring

From the 50th percentile model, observed RULA score is 4 